\title{
Correction to: Microbial spectrum, antibiotic susceptibility profile, and biofilm formation of diabetic foot infections (2014-18): a retrospective multicenter analysis
}

\author{
Fakhria A. Al-Joufi' ${ }^{1}$ Khalid M. Aljarallah ${ }^{2,3} \cdot$ Soheir A. Hagras ${ }^{4,5} \cdot$ Ibrahim M. Al Hosiny $^{6} \cdot$ Mounir M. Salem-Bekhit $^{7}$. \\ Abdullah M. E. Youssof ${ }^{7} \cdot$ Faiyaz Shakeel $^{7}$
}

Published online: 30 August 2021

(c) King Abdulaziz City for Science and Technology 2021

\section{Correction to: 3 Biotech (2020) 10:325 \\ https://doi.org/10.1007/s13205-020-02318-x}

In the original article, the affiliation of Fakhria A. Al-Joufi has been published incorrectly. The correct affiliation should be "Department of Pharmacology, College of Pharmacy, Jouf University, Aljouf 72341, Saudi Arabia".

The authors apologize for this error.

The original article can be found online at https://doi.org/10.1007/ s13205-020-02318-x.

Faiyaz Shakeel

faiyazs@fastmail.fm; fsahmad@ksu.edu.sa

1 Department of Pharmacology, College of Pharmacy, Jouf University, Aljouf 72341, Saudi Arabia

2 College of Applied Medical Sciences, Majmaah University, Majmaah, Saudi Arabia

3 College of Applied Sciences, Almaarefa University, Riyadh, Saudi Arabia

4 Inaya Medical Colleges, Riyadh, Saudi Arabia

5 NCRRT, EAEA, Cairo, Egypt

6 Microbiology and Immunology Department, Faculty of Medicine, Al-Azhar University, Cairo, Egypt

7 Department of Pharmaceutics, College of Pharmacy, King Saud University, P.O. Box 2457, Riyadh 11451, Saudi Arabia 